\title{
Rat spatial memory: Resistance to retroactive interference at long retention intervals
}

\author{
WILLIAM W. BEATTY and DAVID A. SHAVALIA \\ North Dakota State University, Fargo, North Dakota 58105
}

\begin{abstract}
An attempt was made to disrupt memory for spatial information by interpolating a task of high similarity to the to-be-remembered task during a long retention interval. Rats were trained in an 8-arm maze in which choosing each arm without repetition was the optional strategy. A 4-h delay was imposed between the 4 th and 5 th choices. At various times during the retention interval, the rats ran a second identical maze located in another room. No evidence of retroactive interference was observed. In the second experiment, the rat was required to remember the interpolated spatial task during the retention test. This was accomplished by allowing the rat to make four choices in the first maze and then, after a variable period of time, four choices in the second maze. Four hours after exposure to each maze, retention was tested. Choice accuracy on the retention tests was high and equivalent on both mazes. Requiring the rat to remember which arms it had visited in a second maze did not impair memory for the first maze. These results demonstrate that rats can segregate spatial memories established in different contexts with considerable proficiency.
\end{abstract}

When rats are rewarded for adopting a win-shift food-searching strategy in a radial maze, they exhibit highly accurate memory for spatial location (Olton, 1978; Olton \& Samuelson, 1976). In addition to being highly accurate, rat spatial memory is quite longlasting. Beatty and Shavalia (1980) imposed a delay between the 4th and 5th choices in an 8-arm maze and found that choice accuracy exceeded $90 \%$ on Choices 5-8 for delays as long as $4 \mathrm{~h}$. Memory declined at longer retention intervals but exceeded chance at delays as long as $12 \mathrm{~h}$.

Another intriguing property of rat spatial memory is its apparent resistance to retroactive interference (RI). Maki, Brokofsky, and Berg (1979) attempted to produce RI by exposing rats to various visual, auditory, or olfactory stimuli, or forcing them to consume food or perform in another spatial task (a 4-arm maze), during a 2-min retention interval. None of these treatments affected spatial memory, but it could be argued that their use of a short retention interval, which is well below the limits of the rat's spatial memory span, provided an insensitive test of memory disruption because performance was at ceiling.

Recently, we (Shavalia \& Beatty, Note 1) have shown that administration of electroconvulsive shock (ECS) during a 4-6-h retention interval imposed between the 4th and 5th choices disrupts the rat's memory for arms it has visited in an 8-arm maze. The

We thank William S. Maki for his helpful advice throughout this research. amnesic effect of ECS was clearly time-dependent. ECS disrupted memory only if it was administered at least $30 \mathrm{~min}$ after the 4th choice. When given 0 or 15 min after Choice 4, ECS was ineffective. A similar time-dependent effect of an amnesic treatment has been demonstrated by Roberts and Grant (1978), who observed that the disruptive effects of light on matching-to-sample performance by pigeons were greatest if the houselights were turned on late in the retention interval. Thus, Maki et al. (1979) may also have failed to find evidence of RI in rat spatial memory because they administered their interfering events at the wrong time.

In the present experiments, we attempted to maximize the chance of observing RI by using a long retention interval and an interpolated task that was very similar to the event that was to be remembered. Furthermore, since the influence of amnesic treatments may depend upon when they are introduced, we inserted the interpolated task at several different times during the retention interval.

\section{EXPERIMENT 1}

\section{Method}

Subjects. The animals were 11 male rats obtained from the Holtzman Co. (Madison, Wisconsin) when they were about 3 months old. Prior to the present study, they had received more than 6 months of training in one of the mazes used in the present study. Each of the animals had also received eight ECS treatments (.39 W-sec each), which produced the effects on spatial memory described above (Shavalia \& Beatty, Note 1). At the time of the present experiment, the rats were about 10 months old.

During the present studies, the rats were housed singly in an air- 
conditioned animal room that was illuminated from 0700 to $1900 \mathrm{~h}$. They were maintained on a restricted feeding schedule (Purina Lab Chow pellets) designed to maintain their body weights at $85 \%$ of free-feeding levels. Water was always available in the home cage. Behavioral tests occurred during the light portion of the light-dark cycle.

Apparatus. Two 8-arm mazes, as nearly identical as possible, were employed. One of the mazes and the test room that housed it (Maze B) were identical to those used by Maki et al. (1979), except for the addition of black plastic sidewalls $(3.5 \mathrm{~cm}$ high) that extended the length of each arm. The other maze (Maze A) was identical in design but was housed in a somewhat larger room than Maze B. This room was also cluttered with surplus apparatus and equipment, including two shuttleboxes, several relay racks, tables, and chairs, and a file cabinet. The rooms housing the two mazes were located at opposite corners of the laboratory; each was approximately $10 \mathrm{~m}$ from the animal room.

Procedure. Prior to the present study, the animals had received extensive training in Maze $A$ and had mastered the win-shift strategy required by the fact that each of the 8 arms was baited with a single $190-\mathrm{mg}$ Noyes pellet only at the outset of each daily test. The animals were free to enter any of the 8 arms at any time, but in fact they always entered 4 different arms during the first 4 choices. They were thoroughly accustomed to the practice of having a delay imposed between the 4 th and 5 th choices by being returned to their home cages, and they consistently maintained about $90 \%$ accuracy on Choices 5-8 at delays of 4-6 $\mathrm{h}$.

Pretraining consisted of habituating the rats to Maze B. The arms were baited in the same manner as for Maze A. Testing continued until they had entered 7 different arms in the first 8 choices and all 8 arms in the first 10 choices for 5 consecutive days. No delay was imposed during these tests. Once the rats met criterion in Maze B, they were given 5 days of additional testing on Maze A with a 4-h delay imposed between the 4th and 5 th choices.

Formal testing commenced on the following day. Each test day began with the rat's being allowed to choose 4 alleys in Maze A. On alternate days, this experience was followed by a test in Maze B, which began $0,15,30,60,120$, or 230 min after the 4 th choice in Maze A had been made (i.e., Choice 4-Maze B interval). Testing in Maze B continued until the rat had entered all 8 arms and consumed the food. Four hours after the 4th choice in Maze A, the rat was tested for retention in Maze A. Only the arms it had not entered were baited with food. The order of times that the rats were introduced to Maze B (i.e., Choice 4-Maze B intervals) was counterbalanced across subjects, and each rat was tested twice at each temporal interval. On control days, the rats were tested in Maze A with the same 4-h delay imposed between the 4th and 5th choices, but they were not exposed to Maze B. Except when they were actually being tested in the mazes, the rats were housed in their home cages in the animal room. Each time the rat was placed into the center of the maze for a test, it was oriented with its head away from the doorway to the test room. Since the rats usually turned around several times before the doors were raised, this practice was probably unimportant.

\section{Results}

Table 1 displays the results of the retention tests. Despite the interpolation of testing in Maze $B$ at various times after exposure to the to-be-remembered event in Maze A, working memory was consistently accurate. Somewhat surprisingly a repeated measures analysis of variance revealed a significant treatment effect $[F(6,60)=2.96, p<.05]$. Subsequent analysis with Dunnet's test demonstrated reliable differences between the 0 -min condition and the control and between the 30-min condition and the control $(\mathrm{p}<.05)$.
Table 1

Percent Correct on Choices 5-8 in Experiment 1

\begin{tabular}{ccc}
\hline $\begin{array}{c}\text { Choice 4-Maze B } \\
\text { Interval (Minutes) }\end{array}$ & Mean & SEM \\
\hline Control & 92.8 & 1.6 \\
0 & 97.8 & 1.5 \\
15 & 94.5 & 2.6 \\
30 & 87.7 & 2.9 \\
60 & 91.3 & 1.7 \\
120 & 87.8 & 3.3 \\
230 & 90.0 & 3.7 \\
\hline
\end{tabular}

Differences between other temporal conditions and the control condition were not reliable. Performance on Maze B was also accurate; the rats entered an average of 7.8 different arms in the first 8 choices.

It is difficult to imagine why testing in Maze B immediately after exposure to Maze A would improve memory while delaying this treatment by $30 \mathrm{~min}$ would have the opposite effect, so we assume these differences reflect sampling error which attained statistical significance because there is little variability in rats' 8-arm maze performance.

\section{EXPERIMENT 2}

Working memory for spatial information survived exposure to a very similar spatial task when it was interpolated at various times during the delay interval. This demonstrates that rats can segregate contextually distinct spatial information in memory, even when they must remember some of the information for several hours. However, the interpolated spatial task used in Experiment 1 required $10 \mathrm{~min}$ or less for the animal to perform; once completed, the rat did not have to retain it. We reasoned that requiring the animals to remember the interpolated as well as the original spatial task might provide them with a more challenging assignment, thus permitting a more vigorous assault on working memory.

\section{Method}

The subjects, apparatus, and general maintenance procedures were identical to those employed in Experiment 1. As in Experiment 1, two identical 8-arm mazes, housed in different rooms, were used. Before the start of formal testing each rat was trained in Maze B with a 4-h delay imposed between the 4th and 5th choices, during which the rat was confined to its home cage. Such pretraining continued until each rat had entered 7 different arms in the first 8 choices and all 8 arms in the first 10 choices for 5 consecutive days.

During the actual experiment, days on which the rat was exposed to interpolated training were alternated with control days, as in Experiment 1 . The interpolated training occurred as follows. First, the rats were allowed to choose 4 arms in Maze 1 . After a variable interval $(0,15,30,60,120$, or $230 \mathrm{~min})$, they made 4 choices in Maze 2. Four hours after they had made the 4th choice in Maze 1, they were tested for retention in that maze, and $4 \mathrm{~h}$ after they had 
made the 4th choice in Maze 2, they received a retention test in that maze. On control days, the animals were tested only in Maze 1 with a 4-h delay imposed between the 4 th and 5 th choices. The experiment was run in two replications. During the first replication, Maze A served as Maze 1 and Maze B served as Maze 2. The order of the temporal intervals was counterbalanced. The second replication was identical, except that Maze B served as Maze 1 and Maze A served as Maze 2. Thus, the rats received two tests at each of the temporal intervals.

\section{Results}

As shown in Table 2, memory remained quite accurate in all conditions. The rats remembered Maze 1 and Maze 2 equally well; moreover, requiring them to remember which arms they had visited in Maze 2 did not affect their working memory for the spatial information they had experienced in Maze 1 (all Fs $<1.47$ ).

Over the course of their extended training, 4 of the 11 rats developed the habit of visiting 4 adjacent arms on the first 4 choices. No other patterns were evident. Choosing 4 adjacent arms might simplify the memory task since, in principle, the rat might have only to remember to avoid or approach some prominent cue in each maze to maintain high levels of performance. Accordingly, for each animal we determined the percentage of test sessions during Experiment 2 that it entered 4 adjacent arms during the first 4 choices and correlated this value with the animal's memory performance on Choices 5-8. The percentage of days on which the rats chose adjacent arms on Choices 1-4 ranged from 0 to 97 , and average percent correct on Choices 5-8 for Mazes 1 and 2 combined ranged from 73 to 99 . The rank order correlation between these measures was .17. Thus accuracy of memory in the 8-arm maze seems only weakly related to the idiosyncratic response patterns that some animals develop.

This result is not.unexpected. In earlier work, Beatty and Shavalia (1980) demonstrated that spatial memory over a 4-h delay was equally accurate if the rat made the first 4 choices in whatever order it wished or followed a programmed schedule of choices designed to prevent response patterning and the use of landmark strategies.

\section{DISCUSSION}

In the present experiments, we attempted to maximize the opportunity to observe RI by using a long retention interval and an interpolated task of high similarity to the to-be-remembered event. Spatial memory remained intact, even if the rats had to remember both the original spatial task and the interpolated problem. Our findings extend the earlier demonstration that spatial memory in rats is quite resistant to RI (Maki et al., 1979). These workers suggested that rats may be capable "of processing spatial memories established in different contexts in parallel and without loss" (Maki et al., 1979, p. 30).
Table 2

Percent Correct on Choices 5-8 in Experiment 2

\begin{tabular}{ccccc}
\hline \multirow{2}{*}{$\begin{array}{c}\text { Maze 1-Maze 2 } \\
\text { Interval (Minutes) }\end{array}$} & Mean & SEM & Mean & SEM \\
\cline { 2 - 5 } Control & 90.5 & 2.0 & & \\
0 & 88.9 & 3.5 & 87.7 & 3.6 \\
15 & 86.5 & 4.5 & 88.9 & 2.6 \\
30 & 87.7 & 2.9 & 90.0 & 3.7 \\
60 & 90.0 & 3.7 & 87.6 & 4.1 \\
120 & 85.5 & 2.9 & 92.2 & 3.0 \\
230 & 90.5 & 4.4 & 90.0 & 4.3 \\
\hline
\end{tabular}

Our findings are consistent with that proposition, demonstrating that rats can remember spatial locations in two identical 8-arm mazes just as well as they can retain spatial information from one such maze. Since intramaze cues are ineffective in guiding choice behavior in the radial maze (Olton \& Collison, 1979), differential extramaze cues as well as the differing spatial locations of the test rooms presumably allowed the animals to segregate these otherwise similar spatial memories.

This interpretation must remain tentative, of course, since we have no data examining retention performance of animals tested in two identical rooms. Based on Olton's demonstration that proactive interference builds up over repeated tests in the same maze and test room (see Olton, 1978), it might be expected that RI would be more powerful if both intra- and extramaze cues were quite similar.

\section{REFERENCE NOTE}

1. Shavalia, D. A., \& Beatty, W. W. Effects of ECS on spatial memory in rats. Manuscript in preparation.

\section{REFERENCES}

Beatty, W. W., \& Shavalia, D. A. Spatial memory in rats: Time course of working memory and effect of anesthetics. Behavioral and Neural Biology, 1980, 28, 454-462.

MAKI, W. S., Brokofsky, S., \& Berg, B. Spatial memory in rats: Resistance to retroactive interference. Animal Learning \& Behavior, 1979, 7, 25-30.

Olton, D. S. Characteristics of spatial memory. In S. H. Hulse, H. Fowler, \& W. K. Honig (Eds.), Cognitive processes in animal behavior. Hillsdale, N.J: Erlbaum, 1978.

Olton, D. S., \& Collison, C. Intramaze cues and "odor trails" fail to direct choice behavior on an elevated maze. Animal Learning \& Behavior, 1979, 7, 221-223.

Olton, D. S., \& Samuelson, R. J. Remembrance of places passed: Spatial memory in rats. Journal of Experimental Psychology: Animal Behavior Processes, 1976, 2, 97-116.

Roberts, W. A., \& Grant, D. S. An analysis of light-induced retroactive inhibition in pigeon short-term memory. Journal of Experimental Psychology: Animal Behavior Processes, 1978, 4, 219-236.

(Received for publication March 24, 1980; revision accepted June 24,1980 .) 